Reprod. Nutr. Dévelop., 1986, 26 (2 B), 677-682.

\title{
Influence de l'ingestion de lactose sur le métabolisme protéique de l'agneau nouveau-né
}

\author{
P. PATUREAU-MIRAND, E. DEBRAS, J. PRUGNAUD
}

avec la collaboration technique de M. L. HOULIER, M. SALLAS, A. SELLE et M. C. VALLUY

Laboratoire d'Etude du Métabolisme azoté

I.N.R.A. Theix, 63122 Ceyrat, France.

\section{Summary. Effects of lactose feeding on protein metabolism of newborn lambs.}

Whole body and tissue protein turnovers were measured in 6 newborn lambs taken from their mothers immediately after birth. Three lambs (AJ) were hourly fed $50 \mathrm{ml}$ of saline $(\mathrm{NaCl} 0.9 \%)$, and $3(\mathrm{AL})$ were fed, on the same schedule, $50 \mathrm{ml}$ of saline with $2.25 \mathrm{~g}$ of lactose added. They were continuously infused L- $\left[4,5^{3} \mathrm{H}\right]$-leucine for $6 \mathrm{~h}$ when they were $2 \mathrm{~h} 30 \mathrm{~min}$ old. Plasma glucose and insulin were higher in AL than in AJ lambs. On the contrary, the lowest plasma levels of free threonine, valine, isoleucine, leucine, phenylalanine, lysine, histidine, serine and alanine occurred in the lactose-fed lambs (table 1). The concentrations of most free amino acids in liver, brain, small intestine and muscle (Longissimus dorsi) were not significantly different (fig. 1). The irreversible loss of plasma leucine did not differ (mean $\pm S D: 160 \pm 47$ and $156 \pm 11 \mu \mathrm{moles} / \mathrm{h} / \mathrm{kg}$ for $\mathrm{AL}$ and AJ lambs, respectively). The leucine catabolic rate was higher in AJ than in AL lambs $(22.4 \pm 2.8$ vs $17.9 \pm 1.7 \%)$. The fractional rates of protein synthesis in the liver, small intestine and brain were not significantly different between $A L$ and $A J$ lambs; these rates were higher in the muscle, lungs and whole body of the AL lambs (table 2).

\section{Introduction.}

L'ingestion de colostrum s'accompagne d'une stimulation du métabolisme protéique de l'agneau nouveau-né qui se manifeste par un accroissement des échanges d'acides aminés entre le plasma et les tissus, de leur catabolisme et de l'activité de la protéosynthèse dans le muscle (Patureau-Mirand et al., 1985a). La digestion du lactose du colostrum assure au nouveau-né, dès les premiers repas, un apport de glucose exogène qui a un effet insulino-sécréteur chez le fœtus ovin (Philipps et al., 1978) et chez l'agneau nouveau-né (Philipps, Dubin et Raye, 1981). Or, l'insuline favorise l'incorporation des acides aminés dans les protéines musculaires (Goldberg et al., 1980). Ainsi l'augmentation de la protéosynthèse musculaire consécutive à l'ingestion de colostrum par l'agneau nouveau-né pourrait en partie résulter des effets de la digestion du lactose. Afin de vérifier cette hypothèse, le métabolisme protéique d'agneaux nouveau-nés à jeun ou alimentés à l'aide d'une solution de lactose, a été étudié au cours des 9 premières heures après la naissance, selon la méthode de perfusion continue d'un acide 
aminé marqué, décrite et analysée dans l'ouvrage de Waterlow, Garlick et Millward (1978).

\section{Matériel et méthodes.}

Animaux et alimentation. - Six agneaux mâles de race limousine ou croisés lle de France $\times$ Romanov $\times$ Limousin (IRL) pesant entre $2,8 \mathrm{~kg}$ et $3,5 \mathrm{~kg}$ sont séparés de leur mère dès leur naissance. Ils sont installés dans une cage placée dans un local ventilé ayant une température constante de $21^{\circ} \mathrm{C}$ et une hygrométrie voisine de $70 \%$. Un cathéter est alors introduit sous anesthésie locale dans chaque veine jugulaire externe. Dès $1 \mathrm{~h}$ après leur naissance, ces 6 agneaux, répartis en 2 lots ( 2 IRL et 1 Limousin), sont alimentés toutes les heures au biberon. Les uns

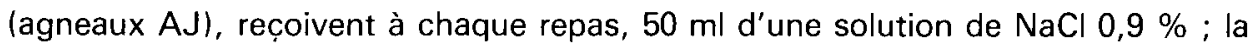
même solution additionnée de $2,25 \mathrm{~g}$ de lactose, est distribuée aux 3 autres (agneaux $A L$ ), de façon à leur apporter une quantité de lactose proche de celle fournie par $50 \mathrm{ml}$ de colostrum.

Perfusion et prélèvements. - 2 h 30 min après leur naissance, les agneaux sont soumis à une perfusion continue de L-leucine ${ }^{3} \mathrm{H}-4,5$ (activité spécifique : $1 \mathrm{Ci} / \mathrm{mmole}$; Amersham), pendant $6 \mathrm{~h}$ environ à raison de $0,28 \mathrm{mCi} / \mathrm{h}$. Des prélèvements de sang jugulaire sont effectués $2 \mathrm{~h}, 4 \mathrm{~h}$ et $5 \mathrm{~h} 45 \mathrm{~min}$ après le début de la perfusion. En fin de perfusion, les agneaux sont sacrifiés par égorgement après anesthésie générale au pentobarbital sodique. Le foie, le muscle long dorsal, le cerveau, les poumons et un échantillon moyen d'intestin grêle sont rapidement prélevés et congelés dans l'azote liquide avec le reste du corps.

Analyse et calculs. - Les teneurs plasmatiques en glucose sont déterminées par la méthode à la glucose-oxydase ; celles de l'insuline et du cortisol, par radioimmunologie au moyen des trousses CEA-SORIN-INSIK 1 et CORTCTK-125 respectivement. Les concentrations en acides aminés libres du plasma et des tissus ainsi que celles des protéines tissulaires, sont mesurées à l'aide des méthodes décrites précédemment (Patureau-Mirand et al., 1985b). La radioactivité de la leucine libre des tissus ou des protéines a été mesurée à l'aide d'un compteur à scintillation liquide (460 CD, Packard) après séparation de la leucine par chromatographie sur la colonne de résine échangeuse de cations d'un analyseur d'acides aminés (Carlo-Erba). Les méthodes utilisées pour le calcul du flux apparent de leucine lou perte irréversible de leucine plasmatique), de son taux de clairance métabolique, du taux et du flux de catabolisme de la leucine ainsi que pour l'estimation du taux de renouvellement (ou de synthèse) des protéines dans les tissus (Ksi), ont été explicitées précédemment (Patureau-Mirand et al., 1985a). La comparaison des concentrations des différents constituants plasmatiques analysés, est réalisée par analyse de variance de façon à pouvoir distinguer un effet âge d'un effet régime (Snedecor et Cochran, 1971).

\section{Résultats et discussion.}

Composés plasmatiques et acides aminés libres tissulaires (tabl. 1). - La glycémie des agneaux AJ reste basse pendant toute la période étudiée alors que 
celle des agneaux $A L$ est élevée dès le premier prélèvement ( 3 h 30 min après le premier repas) et très nettement supérieure à celle d'agneaux du même âge nourris avec du colostrum (Patureau-Mirand et al., 1985b). L'insulinémie des agneaux recevant le lactose est légèrement plus élevée (22\%) que celle des agneaux $\mathrm{AJ}$; ces valeurs sont cependant inférieures à celles observées chez des agneaux nouveau-nés nourris par leur mère (Grizard, Patureau-Mirand et Tissier, 1979) ou soumis à une perfusion de glucose conduisant à une glycémie comparable à celle des agneaux AL (Philipps, Dubin et Raye, 1981). L'ingestion de lactose semble donc avoir un effet sur l'insulinémie moins marqué que celui de la perfusion de glucose ou de l'ingestion de colostrum qui, dans ce dernier cas, peut aussi résulter de l'action insulino-sécrétrice des acides aminés alimentaires. Les teneurs plasmatiques en cortisol, mesurées pour préciser le niveau plasmatique de cette hormone qui réduit l'activité de la protéosynthèse et stimule le catabolisme protéique dans le muscle (Millward et al., 1983), sont élevées et présentent une grande variabilité ; elles sont comparables à celles rapportées par Bosc et Fèvre (1977) pour des animaux de même âge et ne sont pas différentes dans les 2 lots. Les teneurs en acides aminés libres du plasma évoluent peu entre $4 \mathrm{~h} 30$ et $8 \mathrm{~h} 15 \mathrm{~min}$ après la naissance; les valeurs observées chez les agneaux AJ sont voisines de celles rapportées par Patureau-Mirand et al. (1985b). Chez les agneaux AL, les concentrations plasmatiques en thréonine, valine, isoleucine, leucine, phénylalanine, lysine, histidine, sérine et alanine sont significativement plus faibles que chez les agneaux AJ. Cette baisse des teneurs en acides aminés libres du plasma à la suite de l'ingestion de glucides a déjà été décrite depuis longtemps et interprétée comme la manifestation de l'action de l'insuline dont la sécrétion est stimu-

TABLEAU 1

Teneurs plasmatiques en glucose, insuline, cortisol et acides aminés libres.

\begin{tabular}{|c|c|c|c|c|c|}
\hline \multirow{2}{*}{ 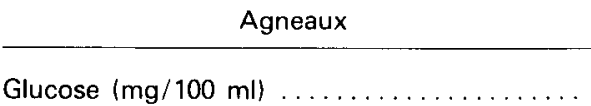 } & \multicolumn{2}{|c|}{ AJ } & \multicolumn{2}{|c|}{$\mathrm{AL}$} & \multirow{2}{*}{$\frac{P(1)}{* *}$} \\
\hline & $28\langle 2\rangle$ & 12 & 176 & 36 & \\
\hline Insuline $(\mu \mathrm{U} / \mathrm{ml}) \ldots \ldots \ldots \ldots \ldots \ldots$ & 19,1 & 0,7 & 24,6 & 4,9 & * \\
\hline $\begin{array}{l}\text { Cortisol }(\mathrm{ng} / \mathrm{ml}) \ldots \ldots \ldots \ldots \ldots \ldots \ldots \\
\text { Acides aminés libres }(\mathrm{mg} / 100 \mathrm{ml})\end{array}$ & 85 & 41 & 62 & 25 & NS \\
\hline Thréonine $\ldots \ldots \ldots \ldots \ldots \ldots \ldots \ldots$ & 1,64 & 0,6 & 0,57 & 0,16 & * \\
\hline Valine $\ldots \ldots \ldots \ldots \ldots \ldots \ldots \ldots \ldots$ & 0,70 & 0,03 & 0,51 & 0,16 & * \\
\hline Isoleucine . & 0,27 & 0,08 & 0,16 & 0,05 & * \\
\hline Leucine ..... & 0,56 & 0,07 & 0,38 & 0,11 & * \\
\hline Phénylalanine .. & 0,70 & 0,10 & 0,57 & 0,08 & * \\
\hline Lysine ........ & 0,44 & 0,11 & 0,30 & 0,10 & * \\
\hline Histidine ..... & 0,93 & 0,15 & 0,69 & 0,08 & ** \\
\hline Arginine $\ldots \ldots \ldots \ldots \ldots \ldots \ldots \ldots \ldots$ & 0,50 & 0,07 & 0,56 & 0,13 & NS \\
\hline Sérine $\ldots \ldots$ & 2,13 & 0,63 & 1,09 & 0,23 & * \\
\hline Glycine ..... & 3,33 & 0,33 & 3,45 & 0,48 & NS \\
\hline Alanine ... & 2,06 & 0,31 & 1,61 & 0,33 & $*$ \\
\hline Citrulline . . & 0,63 & 0,23 & 0,41 & 0,14 & NS \\
\hline Ornithine ..... & 0,22 & 0,06 & 0,19 & 0,06 & NS \\
\hline
\end{tabular}

(1) Effet lactose dans l'analyse de variance à 2 facteurs (lactose et âge). NS : non significatif, * $P<0,05$, ** $P<0,01$. (2) Moyenne suivie de l'écart-type. 
lée par la digestion des glucides (Munro, 1970). Cet effet de l'ingestion de lactose sur les teneurs en acides aminés libres du plasma ne se retrouve pas nettement dans les tissus (fig. 1). Ainsi dans le foie, seules les concentrations en thréonine, valine, leucine et lysine libres sont plus faibles chez les agneaux $A L$ que chez les agneaux $A J$, les teneurs des autres acides aminés n'étant pas différentes. Dans l'intestin grêle des agneaux $A L$, ce sont les teneurs en thréonine, sérine et leucine qui sont plus faibles alors que celles en valine sont plus élevées. Dans le long dorsal, les concentrations en glycine sont plus élevées chez les agneaux AJ que chez les agneaux $A L$ et il n'y a pas de différence entre les 2 lots au niveau du cerveau.
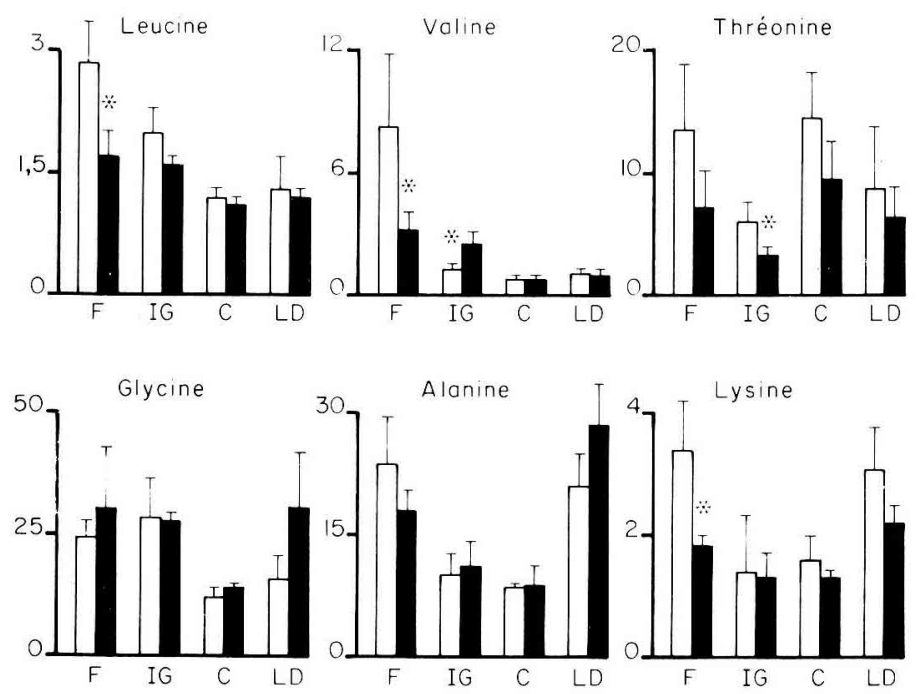

FIG. 1. - Teneurs en acides aminés libres du foie ( $F)$, de l'intestin grêle (/G), du cerveau (C) et du muscle (LD) des agneaux nouveau-nés $A J \square$ ou $A L \square$ (mg p. $100 \mathrm{~g}$ ). ${ }^{*} \mathrm{P}<0,05$.

Flux de leucine. - Le flux plasmatique de leucine n'est pas significativement différent chez les agneaux $A L$ et $A J$ (moyennes \pm écart-types : $160 \pm 47$ et $156 \pm 11$ micromoles par $\mathrm{h}$ et par $\mathrm{kg}$ respectivement). La dispersion des valeurs pour le lot AL est considérable (121 à 213) et ne paraît pas liée au génotype. Le taux de clairance métabolique de la leucine est plus élevé chez les agneaux $A L$ que chez les agneaux AJ $199 \pm 27$ et $65 \pm 1 \mathrm{ml}$ par min et par $\mathrm{kg}$ de poids corporel). Ces flux plasmatiques de la leucine et, dans le cas des agneaux $A J$, son taux de clairance métabolique sont proches des valeurs observées précédemment chez des agneaux nouveau-nés à jeun (Patureau-Mirand et al., 1985a).

Le taux de catabolisme de la leucine est significativement plus faible chez les agneaux $A L$ que chez les agneaux $A J(17,9 \pm 1,7$ et $22,4 \pm 2,8$ p. 100 respectivement). Cependant, du fait de la variabilité observée pour les flux, les quantités de leucine catabolisée ne sont que légèrement plus faibles chez les agneaux $A L$ que chez les agneaux AJ ( 28 et 35 micromoles par h et par $\mathrm{kg}$ respectivement). Les quantités de leucine prélevées dans le plasma et incorporées dans les protéines corporelles (différences entre le flux plasmatique de leucine et son flux de cata- 
bolisme) ne sont pas différentes (132 \pm 42 et $121 \pm 7$ micromoles par h et par $\mathrm{kg}$ respectivement chez les agneaux $A L$ et $A J)$.

Taux de renouvellement des protéines tissulaires. - Les taux de renouvellement des protéines (Ksi), calculés en supposant que la radioactivité spécifique de la leucine dans le compartiment précurseur pour la synthèse protéique est égale à celle de la leucine libre intratissulaire, sont rapportés dans le tableau 2. Dans le foie et l'intestin grêle, ces valeurs sont comparables à celles mesurées chez le fœtus ovin par Schaefer et Krishnamurti (1984) et chez l'agneau âgé d'une semaine (Attaix, Manghebati et Arnal, 1986). Les taux de renouvellement des protéines dans le poumon, le cerveau et le muscle sont plus faibles que ceux observés chez le fœtus ovin en fin de vie fœtale (Horn et al., 1981 ; Schaefer et Krishnamurti, 1984). Dans le poumon et surtout le muscle des agneaux $A L$, les taux de renouvellement des protéines sont significativement plus élevés (58 et $93 \%$ respectivement) que dans ceux des agneaux $\mathrm{AJ}$; cette différence se retrouve au niveau de l'ensemble des protéines corporelles (56\%). Un tel effet de l'apport de glucose ou de glucose et d'insuline sur le taux de renouvellement des protéines tissulaires n'a pas été observé chez le fœetus ovin si ce n'est dans le cerveau (Horn et al., 1981); en revanche, dans le muscle, leur conséquence aurait plutôt été selon ces auteurs, de réduire la protéolyse. Cette dernière ne semble pas avoir été diminuée chez les agneaux $A L$ puisque leurs teneurs musculaires en acides aminés libres ne sont pas différentes de celles des agneaux $\mathrm{AJ}$, bien que la protéosynthèse ait été plus active. La stimulation de cette voie métabolique dans le muscle des agneaux recevant du lactose pourrait en partie résulter de l'action de l'insuline dont les teneurs plasmatiques sont plus élevées que chez les agneaux AJ. Elle peut aussi être la conséquence de l'énergie fournie par le lactose ingéré.

TABLEAU 2

Taux de renouvellement des protéines tissulaires $1 \%$ par jour).

\begin{tabular}{|c|c|c|c|c|c|}
\hline Agneaux & \multicolumn{2}{|c|}{ AJ } & \multicolumn{2}{|c|}{$\mathrm{AL}$} & $P(1)$ \\
\hline Nombre d'animaux & \multicolumn{2}{|c|}{3} & \multicolumn{2}{|c|}{3} & \\
\hline Foie & 68,5 & $10,7(2)$ & 53,1 & $0,8(3)$ & NS \\
\hline$\ldots \ldots \ldots \ldots \ldots \ldots$ & 73,9 & 15,7 & 67,4 & 7,1 & NS \\
\hline$\ldots \ldots \ldots \ldots \ldots \ldots \ldots$ & 33,4 & 1,2 & 52,8 & 2,3 & ** \\
\hline Cerveau & 24,0 & 0,8 & 21,4 & 5,9 & NS \\
\hline Long dorsal .... & 7,2 & 1,0 & 13,9 & 0,5 & $* *$ \\
\hline Corps entier ..... & 17,4 & 0,6 & 27,1 & 4,9 & ** \\
\hline
\end{tabular}

(1) Comparaison à l'aide du test $t$; NS : non significatif ; ** $P<0,01$. (2) Moyenne suivie de l'écart-type (SD). (3) 2 animaux.

\section{Conclusion.}

L'ingestion de lactose a des effets sur le métabolisme protéique de l'agneau nouveau-né (baisse des teneurs plasmatiques en acides aminés libres, stimulation 
de la protéosynthèse musculaire, réduction de la dégradation des acides aminés) qui peuvent suggérer un effet médiateur de l'insuline. Cependant, un effet propre du glucose ou d'autres médiateurs ne peut être exclu. La digestion du lactose du colostrum paraît donc avoir un rôle important dans la stimulation du métabolisme protéique après la naissance.

$11^{e}$ Réunion du groupe Développement I.N.R.A., Montpellier, 22-24 mai 1985.

Remerciements. - Nous remercions vivement M. J. Grizard pour sa participation aux dosages de l'insuline et Mme P. Journaix pour la détermination des concentrations en glucose plasmatique.

\section{Références}

ATTAIX D., MANGHeBATI A., ARNAL M., 1986. Protein synthesis in small intestine and liver during postnatal development in the lamb. Reprod. Nutr. Dévelop., 26, 703-704.

BOSC M.., FĖVRE J., 1977. Evolution de la cortisolémie au cours de la première semaine de vie chez le Veau (Bos taurus) et chez l'Agneau (Ovis aries). C. R. Acad. Sci. Paris, 284, 23732376.

GOLDBERG A. L., TISCHLER M., DE MARTINO G., GRIFFIN G., 1980. Hormonal regulation of protein degradation and synthesis in skeletal muscle. Fed. Proc., 39, 31-36.

GRIZARD J., PATUREAU-MIRAND P., TISSIER M., 1979. Influence du niveau des apports énergétiques pendant la fin de la gestation sur l'insulinémie et l'aminoacidémie des brebis et de leurs agneaux. Ann. Biol. anim. Bioch. Biophys., 19, 199-205.

HORN J., STERN M. D. R., YOUNG M., NOAKES D. E., 1983. Influence of insulin and substrate concentration on protein synthetic rate in fetal tissues. Res. vet. Sci., 35, 35-41.

MILLWARD D. J., BATES P. C., de BENOIST B., BROWN J. G., COX M., HALLIDAY D., ODEDRA B., RENNIE M. J., 1983. Protein turnover : the nature of the phenomenon and its physiological regulation. Vie Symp. int. Métabolisme et Nutrition azotés, Vol. 1, 69-96. I.N.R.A. Publ. Versailles.

MUNRO H. N., 1970. General aspects of the regulation of protein metabolism by diet and by hormones. In H. N. MUNRO, Mammalian protein metabolism, 4, 380-481. Acad. Press, N.-Y.

PATUREAU-MIRAND P., BERNARD O., PRUGNAUD J., ARNAL M., 1985a. Métabolisme protéique de l'agneau nouveau-né. 2. Influence de l'alimentation sur les flux et le taux de renouvellement des protéines. Reprod. Nutr. Dévelop., 25, 1061-1073.

PATUREAU-MIRAND P., BERNARD O., PRUGNAUD J., LEVIEUX D., PION R., 1985b. Métabolisme protéique de l'agneau nouveau-né. 1 . Conséquences de l'ingestion de colostrum sur la glycémie, la gammaglobulinémie et les teneurs en acides aminés libres des tissus. Reprod. Nutr. Dévelop., 25, 1047-1060.

PHILIPPS A. F., CARSON B. S., MESCHIA G., BATTAGLIA F. C., 1978. Insulin secretion in fetal and newborn sheep. Am. J. Physiol., 39, E467-E474.

PHILIPPS A. F., DUBIN J. W., RAYE J. R., 1981. Maturation of early-phase insulin release in the neonatal lamb. Biol. Neonate, 39, 225-231.

SCHAEFER A. L., KRISHNAMURTI C. R., 1984. Whole body and tissue fractional protein synthesis in the ovine fetus in utero. Br. J. Nutr., 52, 359-369.

SNEDECOR G. W., COCHRAN W. G., 1971. Méthodes statistiques A.C.T.A., Paris, 649 p.

WATERLOW J. C., GARLICK P. J., MILLWARD D. J., 1978. Protein turnover in mammalian tissues and in the whole body. North Holland. Amsterdam. 804 p. 